\section{Geteilte Hausarbeit als Ehekiller?}

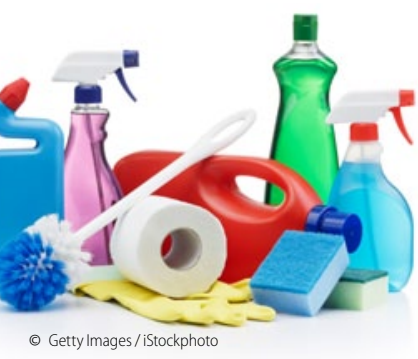

Paare, die sich die Hausarbeit teilen, trennen sich eher als Paare, bei denen die Frau den Großteil der häuslichen Arbeiten übernimmt das zeigt eine norwegische Studie, in dessen Rahmen 18.934 Norweger befragt wurden. Gemeinsames Putzen ist aber nicht direkt schlecht für die Beziehung: Es ist lediglich Indiz dafür, dass die Paare weniger traditionell ausgerichtet sind, die Frauen einen hohen Bildungsgrad haben, finanziell unabhängiger sind, und sie damit auch eine Scheidung besser verkraften.

Hansen Tet al, NOVA Report 8/12 (online first)

\section{Aneurysma-Screening äußerst effektiv}

Bei Männern zwischen 65 und 74 Jahren einmalig per Ultraschall nach einer erweiterten Bauchschlagader zu suchen, senkt die aneurysmabedingte Mortalität, belegt eine britische Studie mit 67.770 Patienten. Diese wurden randomisiert zu einem Screening eingeladen oder nicht. 27.204 Männer (80,3\%) waren der Einladung gefolgt, und bei 1334 war ein abdominelles Aortenaneurysma entdeckt worden. Eine elektive Reparatur wurde in der Einladungsgruppe bei 600 Patienten vorgenommen, gut doppelt so häufig wie in der Kontrollgruppe mit 277 Eingriffen.

Thompson SG et al, Br J Surg 2012 (online first)

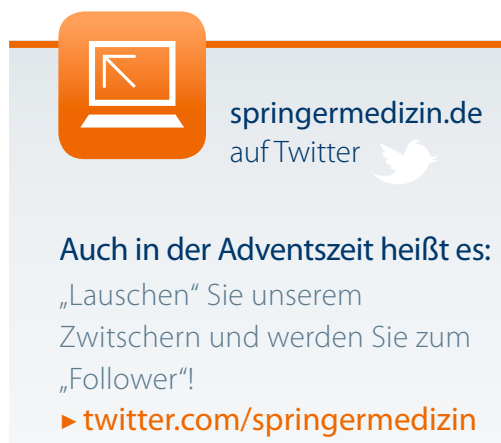

Autoaggression nach Infektion

\title{
Begünstigt Toxoplasmose Selbstmord?
}

Die Ergebnisse einer dänischen Studie stützen die Hypothese, dass Schwangere, die sich mit Toxoplasma gondii infiziert haben, ein erhöhtes Risiko für autoaggressives Verhalten haben, bis hin zum versuchten Suizid. Eine mögliche Erklärung könnte die Überquerung der Blut-Hirn-Schranke von Seiten der lgG-Antikörper sein.

Bereits in zwei früheren Studien gab es Hinweise auf einen Zusammenhang zwischen einer Infektion mit Toxoplasma gondii und Suizidversuchen. Jetzt haben dänische Psychiater diese Assoziation in einer prospektiven Kohortenstudie auf der Basis eines umfangreichen Registers überprüft. Das Follow-up betrug fast 14 Jahre und wertete Informationen von fast 46.000 Frauen aus. Für die Studie wurden die Serumspiegel von IgG-Antikörpern ihrer Neugeborenen gemessen, da die Forscher davon ausgehen, dass die IgG-Werte bei Neugeborenen mit denen der Mütter eng korrelieren. Die IgG-Bestimmung im Blut erfolgte fünf bis zehn Tage nach der Geburt, darüber hinaus bestimmten die Wissenschaftler bei den Frauen die Inzidenz von autoaggressivem Verhalten, Suizidversuchen und Suiziden.

Im Vergleich zu nichtinfizierten Müttern hatten Mütter nach einer Infektion mit Toxoplasmen ein eineinhalbfach erhöhtes relatives Risiko für autoaggressives Verhalten. Das geschätzte relative Risiko für einen Suizidversuch lag bei 1,81 und das für einen Suizid bei 2,05 (Letzteres nicht signifikant). Insgesamt $18 \mathrm{der}$ 45.788 Frauen nahmen sich das Leben. Die Wahrscheinlichkeit für Autoaggressionen war umso höher, je höher die IgGSerumtiter lagen.

(ple)

Pedersen MG et al, Arch Gen Psychiatry 2012, 69(11):1123

\section{Asthma und COPD lauern}

\section{Akuter Husten ist oft nicht harmlos!}

Jeder fünfte Erwachsene, der sich wegen akuten Hustens beim Hausarzt vorstellt, hat Auffälligkeiten in der Spirometrie, die auf ein bisher nicht entdecktes Asthma oder eine nicht erkannte COPD hindeuten.

Nicht nur bei Patienten mit persistierendem, sondern auch mit akutem Husten, sollte an eine chronische Lungenerkrankung gedacht werden. Das verdeutlicht eine Studie, an der Hausärzte aus zwölf europäischen Ländern beteiligt waren. Die Ärzte hatten 1947 Patienten mit einem kürzer als 28 Tage dauernden Husten und ohne COPD- und Asthmadiagnose für die Studie gewinnen können. Die Patienten waren im Mittel 50 Jahre alt, zu 40\% männlich und hatten vor dem Arztbesuch bereits neun Tage gehustet. Vier Wochen nach der Erstvorstellung, wenn der Husten wieder verschwunden war, wurde bei allen Patienten die Lungenfunktion geprüft.

Bei 240 Studienteilnehmern (12\%) fanden sich Hinweise auf ein unerkanntes Asthma. Sie hatten einen positiven Bronchospasmolyse-Test mit einer FEV1-Zunahme (1-Sekunden-Kapazität) um mindestens $12 \%$ und um mehr als $200 \mathrm{ml}$. Patienten unter 50 Jahren waren häufiger betroffen als ältere (15\% vs. 11\%). Anzeichen einer persistierenden Atemwegsobstruktion wurden, je nach Bewertungsmaßstab, bei bis zu 10\% der Patienten entdeckt. (bs)

Van Vugt Set al, Ann Fam Med 2012,10:523 